\title{
A New Class of Wilf-Equivalent Permutations
}

\author{
ZVEZDELINA STANKOVA \\ stankova@mills.edu \\ Department of Mathematics and Computer Science, Mills College, Oakland, CA, USA \\ JULIAN WEST \\ westj@mala.bc.ca; julian@math.uvic.ca \\ Department of Mathematics and Statistics, University of Victoria, Canada
}

Received May 5, 2001; Revised October 9, 2001; Accepted October 17, 2001

\begin{abstract}
For about 10 years, the classification up to Wilf equivalence of permutation patterns was thought completed up to length 6 . In this paper, we establish a new class of Wilf-equivalent permutation patterns, namely, $(n-1, n-2, n, \tau) \sim(n-2, n, n-1, \tau)$ for any $\tau \in S_{n-3}$. In particular, at level $n=6$, this result includes the only missing equivalence $(546213) \sim(465213)$, and for $n=7$ it completes the classification of permutation patterns by settling all remaining cases in $S_{7}$.
\end{abstract}

Keywords: Wilf-equivalent, shape-Wilf-equivalent, restricted patterns, forbidden permutations

\section{Introduction}

A permutation $\tau$ of length $k$ is written as $\left(a_{1}, a_{2}, \ldots, a_{k}\right)$ where $\tau(i)=a_{i}, 1 \leq i \leq k$. For $k<10$ we suppress the commas without causing confusion. As usual, $S_{n}$ denotes the symmetric group on $[n]=\{1,2, \ldots, n\}$.

Definition 1 Let $\tau$ and $\pi$ be two permutations of lengths $k$ and $n$, respectively. We say that $\pi$ is $\tau$-avoiding if there is no subsequence $i_{\tau(1)}, i_{\tau(2)}, \ldots, i_{\tau(k)}$ of $[n]$ such that $\pi\left(i_{1}\right)<\pi\left(i_{2}\right)<\cdots<\pi\left(i_{k}\right)$. If there is such a subsequence, we say that the subsequence $\pi\left(i_{\tau(1)}\right), \pi\left(i_{\tau(2)}\right), \ldots, \pi\left(i_{\tau(k)}\right)$ is of type $\tau$.

For example, the permutation $\omega=(52687431)$ avoids (2413) but does not avoid (3142) because of its subsequence (5283). An equivalent, but perhaps more insightful, definition is the following reformulation in terms of matrices.

Definition 2 Let $\tau \in S_{n}$. The permutation matrix $M(\tau)$ is the $n \times n$ matrix having a 1 in position $(i, \tau(i))$ for $1 \leq i \leq n$, and having 0 elsewhere. ${ }^{1}$ Given two permutation matrices $M$ and $N$, we say that $M$ avoids $N$ if no submatrix of $M$ is identical to $N$.

Note that a permutation matrix $M$ of size $n$ is simply a traversal of an $n \times n$ matrix, i.e. an arrangement of 1's for which there is exactly one 1 in every row and in every column of $M$. It is clear that a permutation $\pi \in S_{n}$ contains a subsequence $\tau \in S_{k}$ if and only if $M(\pi)$ contains $M(\tau)$ as a submatrix. 
Let $S_{n}(\tau)$ denote the set of $\tau$-avoiding permutations in $S_{n}$.

Definition 3 Two permutations $\tau$ and $\sigma$ are called Wilf-equivalent if they are equally restrictive: $\left|S_{n}(\tau)\right|=\left|S_{n}(\sigma)\right|$ for all $n \in \mathbb{N}$. We denote this by $\tau \sim \sigma$. If $\left|S_{k}(\tau)\right|=\left|S_{k}(\sigma)\right|$ for $k \leq n$, then we say that $\tau$ and $\sigma$ are equinumerant up to level $n$.

The basic problem in the theory of forbidden subsequences is to classify all permutations up to Wilf-equivalence. Obviously, if two permutations are Wilf-equivalent, then they must be of the same length. Further, many Wilf-equivalences can be deduced by symmetry arguments within the same $S_{k}$. For instance, if $M(\pi)$ contains $M(\tau)$ as a submatrix, then the transpose matrix $M(\pi)^{t}$ contains $M(\tau)^{t}$. The same is true when simultaneously reflecting both matrices $M(\pi)$ and $M(\tau)$ in either a horizontal or a vertical axis of symmetry. The three operations defined above generate the dihedral group $D_{4}$ acting on the set of permutation matrices in the obvious way. The orbits of $D_{4}$ in $S_{k}$ are called symmetry classes. It is clear that if $\tau$ and $\sigma$ belong to the same symmetry class in $S_{k}$, then $\tau \sim \sigma$. However, Wilf-classes are in general, but apparently rarely, larger than single symmetry classes. This makes the classification of permutations up to Wilf-equivalence a subtle and difficult process.

The first major result in the theory of forbidden subsequences states that (123) (132), and hence $S_{3}$ is one Wilf-class, which combines the two symmetry classes of (123) and (132). At the behest of Wilf, bijections between $S_{n}(123)$ and $S_{n}(132)$ were given by SimionSchmidt [15], Rotem [14], Richards [13], and West [19]. They all prove $\left|S_{n}(123)\right|=c_{n}$, where $c_{n}$ is the $n$th Catalan number. Permutations with forbidden subsequences arise naturally in computer science in connection with sorting problems and strings with forbidden subwords. For example, in [6-7] Knuth shows that $S_{n}(231)$ is the set of stack-sortable permutations (see also [9]), so that $\left|S_{n}(231)\right|$ is the number of binary strings of length $2 n$, in which 0 stands for a "move into a stack" and 1 symbolizes a "move out from the stack".

Numerous problems involving forbidden subsequences have also appeared in algebraic combinatorics. In the late 1980s, it was discovered that the property of avoiding 2143 exactly characterizes the vexillary permutations, i.e. those whose Stanley symmetric function is a Schur function. (See [10] for a good exposition.) Lakshimibai and Sandhya [8] likewise show that $S_{n}(3412,4231)$ is the set of permutations indexing an interesting subclass of Schubert varieties. And Billey and Warrington [3] have very recently defined a class of permutations under 5 restrictions which are related to the Kazhdan-Lusztig polynomials. This all naturally leads to the study and classification of Wilf-classes of permutations of length 4 or more.

The classification of $S_{4}$ turns out to be much more complicated than that of $S_{3}$. It is completed in a series of papers by Stankova and West. They utilize the concept of a generating tree $\mathfrak{T}(\tau)$ of $\tau \in S_{k}$ : the nodes on the $k$ th level of $\mathfrak{T}(\tau)$ are the permutations in $S_{n}(\tau)$, and the descendants of $\pi \in S_{n}(\tau)$ are obtained from $\pi$ by inserting $n+1$ in appropriate places in $\pi$ so that $\tau$ is still avoided. Clearly, the tree isomorphism $\mathfrak{T}(\tau) \simeq \mathfrak{T}(\sigma)$ implies $\tau \sim \sigma$, but the converse is far from true. In [19], West shows $\mathfrak{T}(1234) \simeq \mathfrak{T}(1243) \simeq \mathfrak{T}(2143)$. In [16], Stankova constructs a specific isomorphism $\mathfrak{T}(4132) \cong \mathfrak{T}(3142)$. In [17], she completes the classification of $S_{4}$ by proving $(1234) \sim(4123)$; there she uses a different approach 


\begin{tabular}{|c||c|c|c|c|c|c|c|c|}
\hline \hline$\tau \in S_{4}$ & $S_{6}(\tau)$ & $S_{7}(\tau)$ & $S_{8}(\tau)$ & $S_{9}(\tau)$ & $S_{10}(\tau)$ & $S_{11}(\tau)$ & $S_{12}(\tau)$ & $S_{13}(\tau)$ \\
\hline \hline$(4132)$ & 512 & 2740 & 15485 & 91245 & 555662 & 3475090 & 22214707 & 144640291 \\
\hline$(1234)$ & 513 & 2761 & 15767 & 94359 & 586590 & 3763290 & 24792705 & 167078577 \\
\hline$(1324)$ & 513 & 2762 & 15793 & 94776 & 591950 & 3824112 & 25431452 & 173453058 \\
\hline
\end{tabular}

Figure 1. Classification of $S_{4}$ up to Wilf-equivalence.

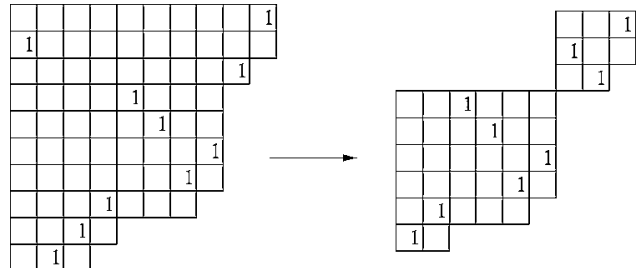

Figure 2. Splitting of a traversal $T \in S_{Y(10,10,9,8,8,8,8,7,4,3)}(213)$.

which yields the somewhat surprising result that, while $\mathfrak{T}(1234) \not \mathfrak{T}(4123)$, on every level of the two trees the number of nodes with a given number of descendants is the same for both trees. Thus, the seven symmetry classes of $S_{4}$ are grouped in three Wilf-classes, with representatives (4132), (1234) and (1324) (cf. figure 1.)

In [1], Babson-West show $(n-1, n, \tau) \sim(n, n-1, \tau)$ for any $\tau \in S_{n-2}$, and $(n-2, n-1$, $n, \tau) \sim(n, n-1, n-2, \tau)$ for any $\tau \in S_{n-3}$, thus completing the classification up to level 5 . The key idea is the concept of a stronger Wilf-equivalence relation.

Definition 4 A traversal $T$ of a Young diagram $Y$ is an arrangement of 1's and 0's such that every row and every column of $Y$ has exactly one 1 in it. A subset of 1 's in $T$ is said to form a submatrix of $Y$ if all columns and rows of $Y$ passing through these 1's intersect inside $Y$. For a permutation $\tau \in S_{k}$, we say that $T$ contains pattern $\tau$ if some $k 1$ 's of $T$ form a submatrix of $Y$ identical to $M(\tau)$ (cf. figure 2.)

Given several 1's in a traversal $T$, the condition for them to form a submatrix of $T$ is the same as the requirement that the column of the rightmost 1 and the row of the lowest 1 must intersect inside $Y$. This condition is necessary for the new definition to be a useful generalization of the classical definition of a forbidden subsequence, as we shall see below. In particular, when $Y$ is a square diagram, the two definitions coincide. Let us denote by $S_{Y}(\tau)$ the set of all traversals of $Y$ which avoid $\tau$.

Definition 5 Two permutations $\tau$ and $\sigma$ are called shape-Wilf-equivalent (SWE) if $\left|S_{Y}(\tau)\right|=\left|S_{Y}(\sigma)\right|$ for all Young diagrams $Y$. We denote this by $\tau \stackrel{s}{\sim} \sigma$.

Clearly, $\tau \stackrel{s}{\sim} \sigma$ implies $\tau \sim \sigma$, but not conversely. We will write $Y\left(a_{1}, a_{2}, \ldots, a_{n}\right)$ for the Young diagram $Y$ whose $i$-th row has $a_{i}$ cells, $1 \leq i \leq n$. In order for a Young diagram $Y$ to have any traversals at all, $Y$ must have the same number of rows and columns and $Y$ must contain the staircase diagram $S t=Y(n, n-1, \ldots, 2,1)$, where $n$ is the number 
of cells in the top (largest) row of $Y$. Thus, from now on, when we talk about a Young diagram $Y$ of size $n$, we will assume that $Y$ has $n$ rows and $n$ columns and contains $S t$ of size $n$.

SWE is a very strong relation on two permutations and it is certainly too restrictive on its own to be useful in the general classification of permutations. However, combined with the proposition below (see [1]), it allows for more Wilf-equivalences to be established.

Proposition 1 Let $A \stackrel{s}{\sim} B$ for some permutation matrices $A$ and $B$. Then for any permutation matrix $C$ :

$$
\left(\begin{array}{l|l}
A & 0 \\
\hline 0 & C
\end{array}\right) \stackrel{s}{\sim}\left(\begin{array}{l|l}
B & 0 \\
\hline 0 & C
\end{array}\right) .
$$

Let $I_{k}$ be the $k \times k$ identity matrix, and let $J_{k}$ be its reflection across a vertical axis of symmetry. According to Backelin-West-Xin in [2], $I_{k} \stackrel{s}{\sim} J_{k}$ for any $k$, and hence $(n, n-$ $1, \ldots, m, \tau) \sim(m, \ldots, n-1, n, \tau)$ for any $\tau \in S_{n-m}$. This SWE generalizes the results in [20] and [1], but it is not sufficient to complete the classification of $S_{7}$, nor of $S_{6}$.

In 2001, Stankova noticed a missing case of a plausible Wilf-equivalence in $S_{6}$ : (546213) and (465213) were equinumerant up to level 11, but no reference was found regarding why these permutations were thought to be in different Wilf classes (see figure 11). Stankova further found an infinite class of Wilf-equivalences

$$
(n-1, n-2, n, \tau) \sim(n-2, n, n-1, \tau),
$$

At her request, West confirmed (1) by computer checks for $n=6,7$ up to level $13 .^{2}$

The purpose of this paper is to explain the proof of the new Wilf-equivalences in (1). The idea is to show (213) $\stackrel{\mathcal{S}}{\sim}(132)$ and apply then Proposition 1. Even though $M(213)$ and $M(132)$ are transposes of each other, their SWE relationship is far from trivial, as the present paper will reveal. It is surprising that such a basic relationship is discovered only now, 10 years after the introduction of SWE in the early 1990s.

Theorem 1 (Main result of the paper) The permutations (213) and (132) are shapeWilf-equivalent. Consequently, for any $\tau \in S_{n-3}$, the permutations $(n-1, n-2, n, \tau)$ and $(n-2, n, n-1, \tau)$ are Wilf-equivalent.

Theorem 1 finally accounts for the last missing case in $S_{6}$ and the remaining cases in $S_{7}$, thus completing the classification of forbidden subsequences up to length $n=7$ (see figure 7).

In summary, modulo symmetry classes, as of now there are essentially two known infinite families of Wilf-equivalences, resulting from [2] and the present paper:

$$
\left(\begin{array}{c|c}
I_{k} & 0 \\
\hline 0 & C
\end{array}\right) \stackrel{s}{\sim}\left(\begin{array}{c|c}
J_{k} & 0 \\
\hline 0 & C
\end{array}\right) \text { and }\left(\begin{array}{c|c}
M(213) & 0 \\
\hline 0 & C
\end{array}\right) \stackrel{s}{\sim}\left(\begin{array}{c|c}
M(132) & 0 \\
\hline 0 & C
\end{array}\right)
$$


Further, there is only one known "sporadic" case of Wilf-equivalence, from [16]:

$$
\left(\begin{array}{llll}
1 & 0 & 0 & 0 \\
0 & 0 & 1 & 0 \\
0 & 0 & 0 & 1 \\
0 & 1 & 0 & 0
\end{array}\right) \sim\left(\begin{array}{llll}
0 & 0 & 1 & 0 \\
1 & 0 & 0 & 0 \\
0 & 0 & 0 & 1 \\
0 & 1 & 0 & 0
\end{array}\right) .
$$

The above (4132) and (3142) in (3) constitute an interesting pair of Wilf-equivalent permutations: (3142) has the smallest symmetry class as it corresponds geometrically to the quadrilateral with most symmetries - the square, while (4132) has the largest symmetry class as it corresponds to the quadrilateral with least number of symmetries-a quadrilateral with 4 different angles. And yet, not only (4132) (3142), but also their trees are isomorphic. In a similar vein, the permutations in (2) are more than just Wilf-equivalentthey are SWE. This is an interesting phenomenon - so far, every known Wilf-equivalence can be explained by a stronger relationship: either symmetry, tree isomorphism, SWE, or a combination of these.

For further discussion, we refer the reader to Sections 5-6.

The proof of the main result (Theorem 1) is structured as follows. Since the permutation matrices of (213) and (132) are transposes, Lemma 2(i)-(ii) in Section 3 allows us to prove the equivalent statement that $Y \equiv Y^{t}$ for all Young diagrams $Y$, i.e. that the number of (213)-avoiding traversals of $Y$ is the same as the number of (213)-avoiding traversals of $Y^{t}$ (cf. Definition 10 in Section 2). To this end, in Section 2 we define an operation on Young diagrams, which we call row-decomposition. It breaks up every Young diagram $Y$ into two smaller diagrams $Y_{r}^{\prime}$ and $Y_{r}^{\prime \prime}$ so that $Y \equiv Y_{r}^{\prime}+Y_{r}^{\prime \prime}$. To link this with the transpose $Y^{t}$, we define an analogous column-decomposition on $Y$, and Lemma 2(iii) shows that establishing $Y \equiv Y_{c}^{\prime}+Y_{c}^{\prime \prime}$ is equivalent to the main result (213) $\stackrel{s}{\sim}(132)$. In Section 4, we investigate a commutativity argument: we apply row decomposition followed by column decomposition, then reverse the order of the two operations and compare the results. This gives us a tool for using induction and the row-decomposition formula to prove the desired column decomposition formula. An amusing consequence of this discussion in presented by Corollary 2 in Section 5.

\section{The row-decomposition formula}

Let $Y$ be a Young diagram with $n$ rows and $n$ columns. We denote by $(k, \ell)$ the intersection cell of row $k$ and column $\ell$, counted from the top left corner of $Y$. A cell in the bottom row of $Y$ is called a bottom cell of $Y$. Let $m$ be the number of bottom cells in $Y$.

Definition 6 For a subset $X$ of cells in $Y$, define the reduction $Y /_{X}$ of $Y$ along $X$ to be the new Young diagram obtained from $Y$ by deleting all rows and columns of $Y$ which intersect $X$.

For example, if the cross of a cell $C$ in $Y$ is the union of the row and column containing $C$, then the reduction $Y /{ }_{C}$ is the diagram obtained from $Y$ by deleting the cross of $C$. The 

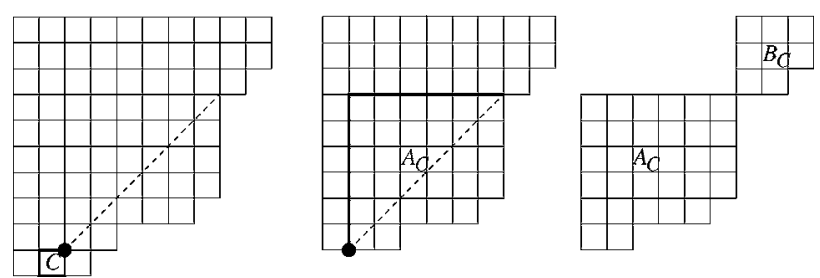

Figure 3. $Y \rightarrow Y /{ }_{C} \rightarrow Y_{C}=A_{C} \times B_{C}$.

reduction of $Y$ along an arbitrary bottom cell of $Y$ is denoted by $Y^{r}$, and it clearly does not depend on the choice of the bottom cell. We will use this fact frequently when reducing along cell $(1, n)$ (and $(n, 1))$ in the proof of the commutativity argument in Lemma 3 in Section 4.

Definition 7 To any bottom cell $C$ in $Y$, we associate a cross-product $Y_{C}$ of Young diagrams in the following way. Mark by $P$ the top right corner of $C$ (this is a grid point on $Y$ ), and consider the reduction $Y /_{C}$, which still contains point $P$. Starting from $P$ in $Y / C$, draw a $45^{\circ}$ ray in north-east direction until the ray intersects for the first time the border of $Y / C$, and use the resulting segment as the south-west/north-east diagonal of a smaller subdiagram $A_{C}$ of $Y /_{C}$. Delete the rows and columns of $A_{C}$ in $Y /_{C}$, leaving a subdiagram $B_{C}=Y /_{\left\{C, A_{C}\right\}}$. Thus, any bottom cell $C$ in the original diagram $Y$ determines a pair $\left(A_{C}, B_{C}\right)$ of smaller Young diagrams, which we call the cross-product of $A_{C}$ and $B_{C}$ and denote by $Y_{C}:=A_{C} \times B_{C}$.

If one of the subdiagrams $A_{C}$ or $B_{C}$ is empty, we define $Y_{C}$ to equal the other subdiagram. This case occurs exactly when $C$ is the first or the last bottom cell of $Y$ :

$$
Y_{(n, 1)}=Y^{r} \times \emptyset=Y^{r}=\emptyset \times Y^{r}=Y_{(n, m)} .
$$

Example 1 Let $Y=Y(10,10,9,8,8,8,8,7,4,3)$. Let $C=(10,2)$. Then $Y_{C}=A_{C} \times$ $B_{C}=Y(6,6,6,6,5,2) \times Y(3,3,2)($ cf. figure 3 .)

Definition 8 Let $Y$ be a Young diagram of size $n$. The row decomposition of $Y$ is the formal sum $\mathcal{R}(Y)$ of cross-products of smaller Young diagrams:

$$
\mathcal{R}(Y):=\sum_{C} Y_{C}=\sum_{C} A_{C} \times B_{C}
$$

where the sum is taken over all bottom cells $C$ of $Y$.

As noted above, the first and the last summands of $\mathcal{R}(Y)$ are identical to $Y^{r}$.

Definition 9 A traversal of a diagram $Y$ which avoids (resp. contains) a (213)-pattern is called a $\operatorname{good}$ (resp. bad) traversal of $Y$. Denote by $\mathcal{T}(Y)=\mathcal{T}\left(a_{1}, a_{2}, \ldots, a_{n}\right)$ the number of good traversals of $Y=Y\left(a_{1}, a_{2}, \ldots, a_{n}\right)$. 
We use the convention $\mathcal{T}(\emptyset)=1$. For some 1 already placed in a cell of $Y$, we say that it imposes a (213)-condition on $Y$ if it plays the role of a " 1 " in a (213)-pattern contained in some bad traversal of $Y$; the (213)-condition is the actual condition on the rest of $Y$ in order to avoid a (213)-pattern containing this 1.

Definition 10 Two diagrams $Y$ and $X$ are said to be numerically equivalent if $\mathcal{T}(Y)=\mathcal{T}(X)$. We denote this by $Y \equiv X$.

Clearly, $\mathcal{T}\left(A_{C} \times B_{C}\right)=\mathcal{T}\left(A_{C}\right) \cdot \mathcal{T}\left(B_{C}\right)$. Moreover, to obtain the number $\mathcal{T}(Y)$, we can apply the function $\mathcal{T}$ to all terms in the formal sum $\mathcal{R}(Y)$ :

Theorem 2 (Row-Decomposition) Let $Y$ be a Young diagram of size n. Then

$$
\mathcal{T}(Y)=\sum_{C} \mathcal{T}\left(A_{C}\right) \cdot \mathcal{T}\left(B_{C}\right)
$$

where the sum is taken over all bottom cells $C$ in $Y$.

Proof: For a bottom cell $C=(n, i)$, let $Y_{i}$ denote the diagram $Y$ with the additional data of 1 in cell $C$. The correspondence will be induced by the map $Y_{i} \mapsto\left(A_{C}, B_{C}\right)$. In fact, we claim that the good traversals of $Y_{i}$ are in 1-1 correspondence with the good traversals of the pair of diagrams $\left(A_{C}, B_{C}\right)$, and hence

$$
\mathcal{T}\left(Y_{i}\right)=\mathcal{T}\left(A_{C}\right) \cdot \mathcal{T}\left(B_{C}\right) .
$$

This, and the fact that any traversal of $Y$ must contain exactly one 1 in the bottom row, immediately establishes Theorem 2. Hence, it suffices to prove (5) for $Y_{i}$.

When $i=1$ or $i=m$, the claim (5) is trivial. Indeed, in these cases, the 1 in the bottom row of $Y$ is either in the first or last bottom cell, and hence it doesn't impose any (213)conditions on $Y$. The question reduces to finding all good traversals of $Y / C=Y^{r}=Y_{C}$. Therefore, $Y_{1} \equiv Y_{m} \equiv Y^{r}$.

Assume now that $1<i<m$. Fix a good traversal $T$ of $Y_{i}$. Denote by $1_{j}$ the 1 in column $j$. By $1_{j}>1_{k}$ we mean that $1_{j}$ is in a row above the row of $1_{k}$. Similarly, for two disjoint sets $A$ and $B$ of 1's, by $A>B$ we mean that all 1's in $A$ are above all 1's in $B$. Let $B_{L}=\left\{1_{1}, 1_{2}, \ldots, 1_{i-1}\right\}$ denote the set of all 1's in $T$ appearing in columns to the left of cell $C$. Similarly, let $A=\left\{1_{i+1}, 1_{i+2}, \ldots, 1_{k}\right\}$ be the set of all 1 's appearing in the columns of $Y$ intersecting $A_{C}$, and let $B_{R}=\left\{1_{k+1}, 1_{k+2}, \ldots, 1_{n}\right\}$ be the set of all 1's appearing in the remaining columns, i.e. all columns to the right of $A_{C}$. Notice that no 1 in $B_{R}$ can appear in a row intersecting $A_{C}$ : the rows of the 1's in $B_{R}$ are above all rows of $A_{C}$ as enforced by the construction of $A_{C}$ via the $45^{\circ}$ segment.

The key idea of the proof is contained in the following lemma:

Lemma 1 In any good traversal $T$ of $Y_{i}$, we have $B_{L}>A$.

Proof (of Lemma 1): Given a row $j$ of $A$, let the level $L_{j}$ be the subset of $A$ consisting of all 1's whose orthogonal projections onto row $j$ are inside $A_{C}$. Clearly, every $1 \in A$ 
belongs to at least one level $L_{j}$, and $L_{n-1} \subseteq L_{n-2} \subseteq \cdots \subseteq L_{n-k}=A$, where $k$ is the size of $A_{C}$. Recall that $i<m$, so that $L_{n-1} \cap A \neq \emptyset$, and that the bottom row of $Y$ is filled by $1_{i}$. This imposes a (213)-condition on $Y_{i}: B_{L}>L_{n-1}$. Finally, from the construction of $A_{C}$, $\left|L_{n-j}\right|>j$ for $j=1,2, \ldots, k-1$, and $\left|L_{n-k}\right|=k$.

We will prove simultaneously the following two statements for all $1 \leq j \leq k$ :

(i) $B_{L}>L_{n-j}$;

(ii) All rows $n-1, n-2, \ldots, n-j$ of $Y_{i}$ are filled in with 1's on level $L_{n-j}$.

For $j=1$, (i) was shown above. But then row $n-1$ in $Y_{i}$ must be filled with an element of $L_{n-1}$, so (ii) is also true. Assume (i) and (ii) for some $j<k$. Then (ii) for $j$, together with $\left|L_{n-j}\right|>j$, implies that at least one element $1_{s}$ of $L_{n-j}$ is in row $n-j-1$ or above. Now (i) for $j$ implies in particular $B_{L}>1_{s}$, so that a new (213)-condition is imposed: $B_{L}>\left(L_{n-j-1} \backslash L_{n-j}\right)$. Combining, $B_{L}>L_{n-j-1}$ : this is (i). By definition of $L_{n-j-1}$, the only $1_{h}$ that can possibly fill in row $n-j-1$ in $Y_{i}$ must belong either to $L_{n-j-1}$, or to $B_{L}$. Because $\left|L_{n-j-1}\right| \geq j+1$ and $B_{L}>L_{n-j-1}$, we conclude that $1_{h} \in L_{n-j-1}$. This shows (ii) for $j+1$ and completes the inductive proof of the above statement. Lemma 1 follows automatically from (i) for $j=k$.

End of Proof of Theorem 2: Combining Lemma 1 with a previous observation, we see that no 1's from $B_{L}$ or from $B_{R}$ can fill the rows intersecting $A_{C}$. In other words, all rows of $A_{C}$ must be filled exactly with the 1's from set $A$ : the number of necessary 1's to make a traversal of $A_{C}$ matches $|A|=k$ because, by construction, $A_{C}$ has as many rows as columns. This in turn forces all 1's in $B_{L}$ and $B_{R}$ to make a good traversal of the subdiagram $B_{C}$. It remains to show that there are no further (213)-conditions imposed by a triple of 1 's coming from $A_{C}$ and $B_{C}$.

The only way for $A_{C}$ and $B_{C}$ to engage together in a (213)-pattern is to have the "2" in $B_{L}$, the " 1 " in $A$, and the " 3 " in $B_{R}$; or, to have the " 2 " and the " 1 " in $A$, and the " 3 " in $B_{R}$. Even though such configurations of three 1's are possible, their "full" matrices will not be contained entirely in $Y$ because of the relative positioning of $A_{C}$ and $B_{R}$.

Putting everything together, the good traversals of $Y_{i}$ are in $1-1$ correspondence with pairs of good traversals of $A_{C}$ and $B_{C}$, i.e. $\mathcal{T}\left(Y_{i}\right)=\mathcal{T}\left(A_{C}\right) \cdot \mathcal{T}\left(B_{C}\right)$ for all bottom cells $C$ of $Y$. This completes the proof of the Row-Decomposition formula.

Example 2 To illustrate the above proof, consider $Y(10,10,9,8,8,8,8,7,4,3)$. Let $Y_{2}$ denote the diagram $Y$ with the additional data that the 1 in the bottom row is in cell $C=(10,2)$. We have to show that $\mathcal{T}\left(Y_{2}\right)=\mathcal{T}(6,6,6,6,5,2) \cdot \mathcal{T}(3,3,2)$ (cf. figure 3.)

The initial condition that $1_{2}$ is in the bottom row forces the (213)-condition $1_{1}>1_{3}$. Since $1_{3}$ is above row 10 , the only 1 's which can fill row 9 are $1_{3}$ and $1_{4}$. If $1_{3}$ is in row 9 , then $1_{1}>1_{4}$ in order to avoid (213); if $1_{4}$ is in row 9, then $1_{1}>1_{3}>1_{4}$. In any case, $1_{1}>1_{3}, 1_{4}$. Without loss of generality, assume that $1_{3}$ is in row 9 , so that $1_{4}$ is in row 8 or above. From $1_{1}>1_{4}$ and avoiding (213), we conclude that $1_{1}>1_{4}, 1_{5}, 1_{6}, 1_{7}$. One of the latter four 1's must fill in row 8 . Without loss of generality, assume that $1_{4}$ is in row 8 ; hence $1_{5}, 1_{6}, 1_{7}$ are in rows 7 or above. But then, to avoid (213), we are forced to conclude that $1_{1}>1_{8}$, i.e. $1_{1}$ is above all of $1_{3}, \ldots, 1_{8}$. We need six 1 's to fill in the six rows $9,8, \ldots, 4$. It 

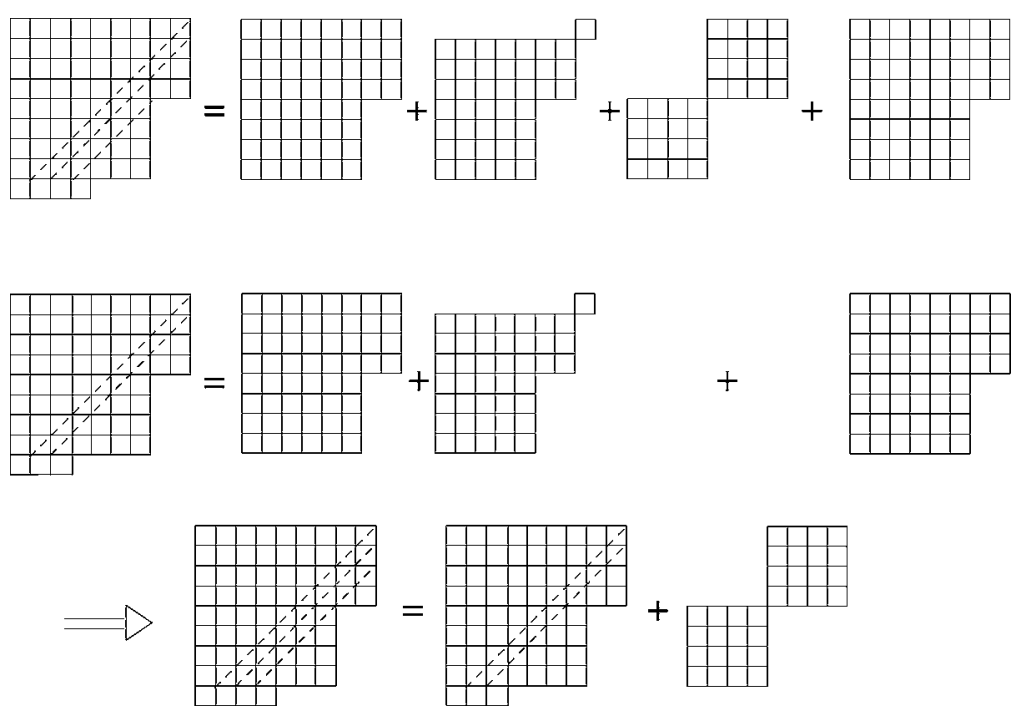

Figure 4. $\mathcal{T}(9,9,9,9,7,7,7,7,4)=\mathcal{T}(9,9,9,9,7,7,7,7,3)+\mathcal{T}(4,4,4,4)^{2}$.

immediately follows that $1_{3}, \ldots, 1_{8}$ must have filled all 6 rows and columns of subdiagram $A_{C}$, leaving all remaining 1's (except for $1_{2}$ ) to form a good traversal of subdiagram $B_{C}$ (cf. figure 2.)

We note that $B_{C}$ consists of two disjoint parts: $B_{L}(1,1,1)$ and $B_{R}(2,2,1)$. The argument that $B_{C}$ and $A_{C}$ cannot engage together in a pattern (213) is identical to the corresponding part of the proof of Theorem 2 .

Given a diagram $Y$, let $C_{b}$ be its rightmost bottom cell, which we call the bottom corner of $Y$, and let $C_{b-1}$ be the bottom cell to the left of $C_{b}$. (In our previous notation, $C_{b}=(n, m)$, $C_{b-1}=(n, m-1)$.) Deleting $C_{b}$ from $Y$ results in a new diagram, which we denote by $Y_{r}^{\prime}$ and call the row-deletion of $Y$. Similarly, we define the right corner $C_{t}$ of $Y$ as the bottom cell in the rightmost column of $Y, C_{t-1}$ as the cell directly above $C_{t}$, and the column-deletion $Y_{c}^{\prime}$ by deleting $C_{t}$ from $Y$. Note that $\left(Y_{r}^{\prime}\right)^{t}=\left(Y^{t}\right)_{c}^{\prime}$, where $X^{t}$ denotes as usual the transpose of diagram $X$ along its main (north-west to south-east) diagonal.

In the row-decomposition of $Y$, we distinguish one special summand: the last but one summand $Y_{C_{b-1}}=A_{C_{b-1}} \times B_{C_{b-1}}$, which we denote by $Y_{r}^{\prime \prime}$.

Corollary 1 For any diagram $Y, Y \equiv Y_{r}^{\prime}+Y_{r}^{\prime \prime}$.

Proof: The row-decomposition of $Y$ includes one more summand than the rowdecomposition of $Y_{r}^{\prime}$, namely, $Y_{r}^{\prime \prime}: \mathcal{R}(Y)=Y_{r}^{\prime}+Y_{r}^{\prime \prime}$. Theorem 2 completes the proof.

From now on, we shall refer to Corollary 1 as Row-Decomposition (RD).

Example 3 Figure 4 illustrates Corollary 1 for $\mathcal{T}(9,9,9,9,7,7,7,7,4)$. 


\section{Column decomposition}

Definition 11 The column decomposition $\mathcal{C}(Y)$ of $Y$ is defined by:

$$
\mathcal{C}(Y)=\sum_{C}\left(\left(Y^{t}\right)_{C^{t}}^{t}\right.
$$

where $C$ runs over all cells in the rightmost column of $Y$, and $C^{t}$ is the image of the cell $C$ after transposing $Y$.

Note that the column decomposition $\mathcal{C}(Y)$ can be obtained directly from $Y$ without going through the transpose $Y^{t}$ : for a cell $C$ in the rightmost column of $Y$, mark the south-west corner of $C$, draw the $45^{\circ}$ ray in south-west direction until it intersects the border of $Y$, delete the cross of $C$ and define analogously the product $Y_{C}=A_{C} \times B_{C}$.

As with row decomposition, we denote by $Y_{c}^{\prime}$ the diagram resulting from $Y$ by deleting the right corner cell $C_{t}$, and by $Y_{c}^{\prime \prime}$ the summand in $\mathcal{C}(Y)$ corresponding to the cell $C_{t-1}$ right above $C_{t}$. By definition, it is clear that

$$
\mathcal{C}(Y)=Y_{c}^{\prime}+Y_{c}^{\prime \prime}
$$

It is not obvious, however, why the same formula should be true after applying the function $\mathcal{T}$ to all terms: why is $Y \equiv Y_{c}^{\prime}+Y_{c}^{\prime \prime}$ ?

Lemma 2 The following statements are equivalent:

(i) $(213) \stackrel{s}{\sim}(132)$

(ii) $Y \equiv Y^{t}$ for all Young diagrams $Y$.

(iii) $Y \equiv Y_{c}^{\prime}+Y_{c}^{\prime \prime}$.

Proof: Let $\mathcal{T}_{\tau}(Y)$ be the number of traversals of $Y$ avoiding permutation $\tau$. In our previous notation, $\mathcal{T}(Y)=\mathcal{T}_{(213)}(Y)$. Since $M(132)=M(213)^{t}, \mathcal{T}_{(132)}(Y)=\mathcal{T}_{(213)}\left(Y^{t}\right)$. By definition of SWE, (i) and (ii) are equivalent.

We will show the equivalence of (ii) and (iii) by induction on the size $n$ of $Y$. When $n \leq 3$, (ii) and (iii) can be easily checked by hand. Note that from the definitions of column reduction, deletion and decomposition, $\left(Y^{t}\right)_{c}^{\prime}=\left(Y_{r}^{\prime}\right)^{t}$ and $\left(Y^{t}\right)_{c}^{\prime \prime}=\left(Y_{r}^{\prime \prime}\right)^{t}$. Assume now that (ii) and (iii) are equivalent for size $\leq n$.

Assume first that (iii) is true for size $\leq n+1$. Then we can use (ii) for sizes $\leq n$ :

$$
Y^{t} \stackrel{(\mathrm{iii})}{\equiv}\left(Y^{t}\right)_{c}^{\prime}+\left(Y^{t}\right)_{c}^{\prime \prime} \stackrel{\text { def }}{=}\left(Y_{r}^{\prime}\right)^{t}+\left(Y_{r}^{\prime \prime}\right)^{t} \stackrel{\text { (ii) }}{=} Y_{r}^{\prime}+Y_{r}^{\prime \prime} \stackrel{\text { RD }}{\equiv} Y .
$$

This shows $Y \equiv Y^{t}$ and completes the proof of (iii) $\Rightarrow$ (ii) for size $n+1$.

Conversely, assume that (ii) is true for size $\leq n+1$.

$$
Y^{t} \stackrel{(\mathrm{ii})}{=} Y \stackrel{\mathrm{RD}}{\equiv} Y_{r}^{\prime}+Y_{r}^{\prime \prime} \stackrel{(\mathrm{ii})}{=}\left(Y_{r}^{\prime}\right)^{t}+\left(Y_{r}^{\prime \prime}\right)^{t} \stackrel{\text { def }}{=}\left(Y^{t}\right)_{c}^{\prime}+\left(Y^{t}\right)_{c}^{\prime \prime}
$$


Replacing $Y$ by $Y^{t}$ in (6), the above reads $Y \equiv Y_{c}^{\prime}+Y_{c}^{\prime \prime}$. This shows (ii) $\Rightarrow$ (iii) for size $n+1$, and completes the proof of (ii) $\Leftrightarrow$ (iii) and of Lemma 2 .

From now on, we shall refer to statement (iii) as Column-Decomposition (CD).

\section{Commutativity of row and column decompositions}

Lemma $3 \quad Y \equiv Y_{c}^{\prime}+Y_{c}^{\prime \prime}$ for all Young diagrams $Y$.

Proof: Assume that the statement is true for all diagrams of size smaller than the size of $Y$. The idea is to apply RD and the assumed CD one after the other in different orders: this results in representing both sides of the equality as sums of the same four terms. Let us start with $Y$. Recall that $C_{b}$ and $C_{t}$ are the bottom and right corners of $Y$, respectively. Apply first RD:

$$
Y \stackrel{\mathrm{RD}}{\equiv} Y_{r}^{\prime}+Y_{r}^{\prime \prime}
$$

Next, apply the assumed CD to $Y_{r}^{\prime}$, and to the factor of $Y_{r}^{\prime \prime}$ that still contains $C_{t}$, leaving the other factor of $Y_{r}^{\prime \prime}$ unchanged:

$$
Y_{r}^{\prime} \stackrel{\mathrm{CD}}{\equiv}\left(Y_{r}^{\prime}\right)_{c}^{\prime}+\left(Y_{r}^{\prime}\right)_{c}^{\prime \prime}, \quad Y_{r}^{\prime \prime} \stackrel{\mathrm{CD}}{\equiv}\left(Y_{r}^{\prime \prime}\right)_{c}^{\prime}+\left(Y_{r}^{\prime \prime}\right)_{c}^{\prime \prime} .
$$

Now start with $Y_{c}^{\prime}+Y_{c}^{\prime \prime}$, and apply RD to $Y_{c}^{\prime}$, and to the factor of $Y_{c}^{\prime \prime}$ that still contains $C_{b}$, leaving the other factor of $Y_{c}^{\prime \prime}$ unchanged:

$$
Y_{c}^{\prime}+Y_{c}^{\prime \prime} \stackrel{\mathrm{RD}}{\equiv}\left(Y_{c}^{\prime}\right)_{r}^{\prime}+\left(Y_{c}^{\prime}\right)_{r}^{\prime \prime}+\left(Y_{c}^{\prime \prime}\right)_{r}^{\prime}+\left(Y_{c}^{\prime \prime}\right)_{r}^{\prime \prime}
$$

In both Eqs. (7) and (8), by abuse of notation, we wrote $\left(Y_{r}^{\prime \prime}\right)_{c}^{\prime},\left(Y_{r}^{\prime \prime}\right)_{c}^{\prime \prime},\left(Y_{c}^{\prime \prime}\right)_{r}^{\prime}$ and $\left(Y_{c}^{\prime \prime}\right)_{r}^{\prime \prime}$ for the cross-products of diagrams resulting from applying $\mathrm{CD}$, resp. $\mathrm{RD}$, to the factors containing $C_{t}$, resp. $C_{b}$. From the definition of row and column deletion, it is clear that the first terms in (7) and (8) are equal: $\left(Y_{r}^{\prime}\right)_{c}^{\prime}=Y-C_{b}-C_{t}=\left(Y_{c}^{\prime}\right)_{r}^{\prime}$. We claim that the remaining three terms also pair up as:

$$
\left(Y_{r}^{\prime}\right)_{c}^{\prime \prime}=\left(Y_{c}^{\prime \prime}\right)_{r}^{\prime}, \quad\left(Y_{r}^{\prime \prime}\right)_{c}^{\prime}=\left(Y_{c}^{\prime}\right)_{r}^{\prime \prime}, \quad\left(Y_{r}^{\prime \prime}\right)_{c}^{\prime \prime}=\left(Y_{c}^{\prime \prime}\right)_{r}^{\prime \prime},
$$

except in Case IV below where

$$
\left(Y_{r}^{\prime}\right)_{c}^{\prime \prime} \equiv\left(Y_{c}^{\prime}\right)_{r}^{\prime \prime}, \quad\left(Y_{r}^{\prime \prime}\right)_{c}^{\prime} \equiv\left(Y_{c}^{\prime \prime}\right)_{r}^{\prime}, \quad\left(Y_{r}^{\prime \prime}\right)_{c}^{\prime \prime} \equiv\left(Y_{c}^{\prime \prime}\right)_{r}^{\prime \prime} .
$$

Before we embark on the proofs of (9-10), note how they fit in the general outline of the proof of CD:

$$
\begin{aligned}
Y & \stackrel{\mathrm{RD}}{\equiv} Y_{r}^{\prime}+Y_{r}^{\prime \prime}(\text { known) } \\
& \stackrel{\mathrm{CD}}{\equiv}\left(Y_{r}^{\prime}\right)_{c}^{\prime}+\left(Y_{r}^{\prime}\right)_{c}^{\prime \prime}+\left(Y_{r}^{\prime \prime}\right)_{c}^{\prime}+\left(Y_{r}^{\prime \prime}\right)_{c}^{\prime \prime} \text { (assumed) } \\
& \equiv\left(Y_{c}^{\prime}\right)_{r}^{\prime}+\left(Y_{c}^{\prime}\right)_{r}^{\prime \prime}+\left(Y_{c}^{\prime \prime}\right)_{r}^{\prime}+\left(Y_{c}^{\prime \prime}\right)_{r}^{\prime \prime} \text { (by examining cases below) } \\
& \equiv Y_{c}^{\prime}+Y_{c}^{\prime \prime} \text { (converse RD, known) }
\end{aligned}
$$


One of the reasons that this works is that the CD-factor in $Y_{r}^{\prime}$ can be anticipated from $\mathcal{B}$, the CD-factor in the original $Y$.

The proof of (9-10) depends solely on how the row and column decomposition interact with each other in any given Young diagram $Y$, more precisely, on the relative position of the two $45^{\circ}$ segments used in the decompositions. Let the $R D$-segment be the segment used in $\mathrm{RD}$, and let the RD-factor be the subdiagram $A_{C}$ determined by the RD-segment, and similarly for $\mathrm{CD}$. Set $\mathcal{A}:=\mathrm{RD}$-factor, and $\mathcal{B}:=\mathrm{CD}$-factor in $Y$. To see that Eqs. (9) and (10) are true, divide all Young diagrams $Y$ into four cases: since the RD- and CD-segments are parallel to each other, there are only four possible relative positions for them. We will use $\stackrel{\text { sym }}{\Rightarrow}, \stackrel{\text { sym }}{=}$ and $\stackrel{\text { sym }}{=}$ as shorthand for "by symmetry arguments".

Case I The RD- and CD-factors do not overlap $(\mathcal{A} \cap \mathcal{B}=\emptyset)$, i.e. the RD- and CD-segments hit $Y$ 's border before they "come close" to each other (figure 12). Then

$$
\begin{aligned}
\left(Y_{r}^{\prime}\right)_{c}^{\prime \prime} & \left.=\left(Y-C_{b}\right)_{c}^{\prime \prime}=\left(Y-C_{b}\right)\right)_{\{(1, n), \mathcal{B}\}} \times \mathcal{B} \\
& =\left(Y /_{\{(1, n), \mathcal{B}\}}\right)_{r}^{\prime} \times \mathcal{B}=\left(Y_{c}^{\prime \prime}\right)_{r}^{\prime} ; \\
\stackrel{\operatorname{sym}}{\Rightarrow}\left(Y_{c}^{\prime}\right)_{r}^{\prime \prime} & \left.=\left(Y-C_{t}\right)\right)_{\{(n, 1), \mathcal{A}\}} \times \mathcal{A}=\left(Y_{r}^{\prime \prime}\right)_{c}^{\prime} ; \\
\left(Y_{r}^{\prime \prime}\right)_{c}^{\prime \prime} & \left.=\left(Y /_{\{(n, 1), \mathcal{A}\}} \times \mathcal{A}\right)_{c}^{\prime \prime}=(Y\}_{\{(n, 1), \mathcal{A}\}}\right)_{c}^{\prime \prime} \times \mathcal{A} \\
& =Y /_{\{(n, 1),(1, n), \mathcal{B}, \mathcal{A}\}} \times \mathcal{B} \times \mathcal{A} \stackrel{\mathrm{sym}}{=}\left(Y_{c}^{\prime \prime}\right)_{r}^{\prime \prime} .
\end{aligned}
$$

Case II The RD-factor contains the $\mathrm{CD}$-factor $(\mathcal{A} \supset \mathcal{B})$, i.e. the RD-segment runs "on the inside" of the CD-segment (see figure 13). As in Case II, $\left(Y_{r}^{\prime}\right)_{c}^{\prime \prime}=\left(Y_{c}^{\prime \prime}\right)_{r}^{\prime}$. Note that $C_{t} \in \mathcal{A}$, and therefore $Y /_{\mathcal{A}}$ is a square. This justifies step (*) below, where $\mathcal{A}^{t r}$ denotes the top right cell of $\mathcal{A}$. Note that $\mathcal{A}^{\text {tr }}$ has the same function in $\mathcal{A}$ as the cell $(1, n)$ has in $Y$. The proof works even in the extreme case where $\mathcal{A}^{\text {tr }}=(1, n)$.

$$
\begin{aligned}
\left(Y_{c}^{\prime}\right)_{r}^{\prime \prime} & =\left(Y-C_{t}\right)_{r}^{\prime \prime}=\left(\mathcal{A}-C_{t}\right) \times Y /_{\{(n, 1), \mathcal{A}\}} \\
& \left.=\mathcal{A}_{c}^{\prime} \times Y /_{\{(n, 1), \mathcal{A}\}}=(\mathcal{A} \times Y\}_{\{(n, 1), \mathcal{A}\}}\right)_{c}^{\prime}=\left(Y_{r}^{\prime \prime}\right)_{c}^{\prime} ; \\
\left(Y_{r}^{\prime \prime}\right)_{c}^{\prime \prime} & \left.=(\mathcal{A} \times Y\}_{\{(n, 1), \mathcal{A}\}}\right)_{c}^{\prime \prime}=\mathcal{A}_{c}^{\prime \prime} \times Y /_{\{(n, 1), \mathcal{A}\}} \\
& \left.=\left.\mathcal{A}\right|_{\left\{\mathcal{B}, \mathcal{A}^{t r}\right\}} \times \mathcal{B} \times Y\right\}_{\{(n, 1), \mathcal{A}\}} ; \\
& \stackrel{*)}{=}\left(Y /_{\{(1, n), \mathcal{B}\}}\right)_{r}^{\prime \prime} \times \mathcal{B}=\left(Y /_{\{(1, n), \mathcal{B}\}} \times \mathcal{B}\right)_{r}^{\prime \prime}=\left(Y_{c}^{\prime \prime}\right)_{r}^{\prime \prime} .
\end{aligned}
$$

Case III The CD-factor contains the RD-factor $(\mathcal{B} \supset \mathcal{A})$, i.e. the $\mathrm{CD}$-segment runs "on the inside" of the RD-segment. This case is symmetric to Case II.

Case IV The RD- and CD-segments overlap (see figure 14). This happens exactly when the RD- and CD-segments differ from each other only in their final cells: the RD-segment intersects the rightmost column of $Y$, while the CD-segment intersects the bottom row of $Y$. Let $\mathcal{D}:=\mathcal{B} / C_{b-1}=\mathcal{A} / C_{t-1}$. Since $\mathcal{B}-C_{b}$ and $\mathcal{A}-C_{t}$ contain exactly one bottom, resp. rightmost, cell, then $\mathcal{B}-C_{b} \equiv \mathcal{D} \equiv \mathcal{A}-C_{t}$ and $\mathcal{B}_{r}^{\prime \prime}=\mathcal{D}=\mathcal{A}_{c}^{\prime \prime}$. Clearly, $\mathcal{S}:=Y /_{\mathcal{D}}$ is a square. Moreover, $\mathcal{S}_{b}=\mathcal{B} / \mathcal{D}=C_{b-1}, \mathcal{S}_{t}=\mathcal{A} / \mathcal{D}=C_{t-1}$; the top right cell of $\mathcal{S}$ is the cell $(1, n)$ of $Y$, and the 
bottom left cell of $\mathcal{S}$ is the cell $(n, 1)$ of $Y$. From this, we see that $Y /\{\mathcal{C},(1, n)\}=Y /\{\mathcal{D},(n, 1)\}$ and $Y /_{\{\mathcal{B},(1, n)\}}=Y /_{\{\mathcal{A},(n, 1)\}}$. Therefore,

$$
\begin{aligned}
\left(Y_{r}^{\prime}\right)_{c}^{\prime \prime} & =\left(Y-C_{b}\right)_{c}^{\prime \prime}=\mathcal{B} / c_{b-1} \times Y /_{\left\{\mathcal{B} / c_{b-1},(1, n)\right\}} \\
& =\mathcal{D} \times Y /\{\mathcal{D},(1, n)\}=\mathcal{D} \times Y /\{\mathcal{D},(n, 1)\} \\
\left(Y_{c}^{\prime \prime}\right)_{r}^{\prime} & =(Y /\{\mathcal{B},(1, n)\} \times \mathcal{B})_{r}^{\prime}=Y /\{\mathcal{B},(1, n)\} \times\left(\mathcal{B}-C_{b}^{\prime}\right)_{r}^{\prime \prime} ; \\
& \equiv Y /\{\mathcal{B},(1, n)\} \times \mathcal{D}=Y /_{\{\mathcal{A},(n, 1)\}} \times \mathcal{D} \stackrel{\text { sym }}{=}\left(Y_{r}^{\prime \prime}\right)_{c}^{\prime} ; \\
\left(Y_{r}^{\prime \prime}\right)_{c}^{\prime \prime} & =\left(\mathcal{A} \times Y /_{\{\mathcal{A},(n, 1)\}}\right)_{c}^{\prime \prime}=\mathcal{A}_{c}^{\prime \prime} \times Y /\{\mathcal{A},(n, 1)\} \\
& =\mathcal{D} \times Y /\{\mathcal{A},(n, 1)\}=\mathcal{D} \times Y /\{\mathcal{B},(1, n)\} \stackrel{\operatorname{sym}}{=}\left(Y_{c}^{\prime \prime}\right)_{r}^{\prime \prime} .
\end{aligned}
$$

The three special subcases when $C_{b-1}=(n, 1)$ (and hence $\left.C_{t-1}=(1, n)\right)$, when $C_{b}=(n, 1)$ (and hence $\left.C_{t}=(1, n)\right)$ and when $C_{b}=C_{t}$ (i.e. $Y$ is a square), are easily checked to satisfy the desired equalities.

The discussion of these four cases completes the proof of Lemma 3.

We remark that, due to the degenerate nature of Case IV, two of the final cross-products turn out to be equal: $\left(Y_{c}^{\prime \prime}\right)_{r}^{\prime}=\left(Y_{c}^{\prime \prime}\right)_{r}^{\prime \prime}$, and hence $\left(Y_{c}^{\prime \prime}\right)_{r}^{\prime \prime}$ has only two factors, rather than the three it has in Cases I-III.

\section{New Wilf equivalences and consequences}

Lemmas 2 and 3 imply the main result of the paper: (213) $\stackrel{s}{\sim}(132)$, which we repeat below as Theorem 1. Combined with Proposition 1, this establishes a new class of Wilf-equivalent permutations.

Theorem 1 The permutations (213) and (132) are shape-Wilf-equivalent. Consequently, for any $\tau \in S_{n-3}$, the permutations $(n-1, n-2, n, \tau)$ and $(n-2, n, n-1, \tau)$ are Wilfequivalent.

In particular, this completes the classification up to Wilf-equivalences of $S_{n}$, for $n \leq 7$. Figure 5 lists the number of symmetry classes and Wilf-classes in each such $S_{n}$.

An amusing corollary about numerical equivalence of Young diagrams can be deduced from the above theorem and the row-decomposition formula. Recall that $S t$ is the standard staircase diagram. The $k$-staircase $S t_{k}$ is the Young diagram which consists of $S t$ plus the full $k-1$ diagonals below the diagonal of $S t$. In particular, $S t$ is $S t_{1}$, and the square $n \times n$ is

\begin{tabular}{|c||c|c|c|c|c|c|c|}
\hline \hline classes & $S_{1}$ & $S_{2}$ & $S_{3}$ & $S_{4}$ & $S_{5}$ & $S_{6}$ & $S_{7}$ \\
\hline \hline symmetry & 1 & 1 & 2 & 7 & 23 & 115 & 694 \\
\hline Wilf & 1 & 1 & 1 & 3 & 16 & 91 & 595 \\
\hline
\end{tabular}

Figure 5. Number of symmetry vs. Wilf classes in $S_{n}, n \leq 7$. 


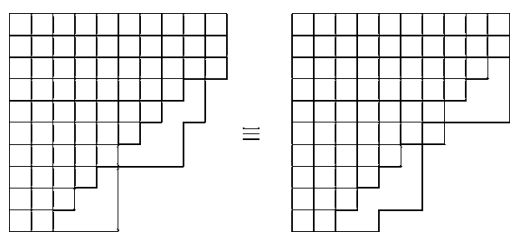

Figure 6. $S t_{2} \cup S t_{2}^{1} \cup S t_{2}^{2} \cup S t_{2}^{3} \equiv S t_{2} \cup\left(S t_{2}^{2}\right)^{t} \cup S t_{2}^{3} \cup S t_{2}^{1}$.

$S t_{n}$. The critical staircase $S t_{k}$ of $Y$ is the first staircase whose complement $Y \backslash S t_{k}$ is a union of at least two connected components. Label such components by $S t_{k}^{j}$, for $j=1,2, \ldots$, starting at the bottom left corner of $Y$. Thus, for every $Y$ with critical staircase $S t_{k}$, we have the critical decomposition $Y=S t_{k} \cup_{j} S t_{k}^{j}$.

Corollary 2 Let $Y=S t_{k} \cup_{j=1}^{\ell} S t_{k}^{j}$ be the critical decomposition of $Y$. The operations of permuting and of transposing the components $S t_{k}^{j}$ result in Young diagrams numerically equivalent to $Y$.

In other words, let $\tau \in S_{\ell}$ be any permutation, and let $\vec{t}=\left(t_{1}, t_{2}, \ldots, t_{\ell}\right) \in\{1, t\}^{\ell}$ correspond to a choice $t_{j}=t$ to transpose, resp. $t_{j}=1$ not to transpose, the component $S t_{k}^{\tau(j)}$. Then the following (ordered) critical decompositions represent numerically equivalent Young diagrams (cf. figure 6):

$$
S t_{k} \bigcup_{j=1}^{\ell} S t_{k}^{j} \equiv S t_{k} \bigcup_{j=1}^{\ell}\left(S t_{k}^{\tau(j)}\right)^{t_{j}}
$$

Proof: We use induction on the size of $Y$. The initial cases are easily verified. Moreover, when there is only one "hanging" shape, the corollary simply states that transposing $Y$ will yield a numerically equivalent diagram $Y^{t}$ : this is the content of Theorem 1 .

Suppose now that there are at least two hanging shapes: $S t_{k}^{1}$ is the bottom shape, and let's name the remaining shapes the "upper" shapes. Choose a permutation $\sigma$ and a transposition vector $\vec{t}$ both of which leave the bottom shape $S t_{k}^{1}$ fixed, and operate on the upper shapes of $Y$. Apply the row-decomposition formula to $Y$ : when $C$ runs over all bottom cells of $Y$, we have

$$
Y \equiv \sum_{C} Y_{C}=\sum_{C} A_{C} \times B_{C}
$$

From the definition of the critical decomposition of $Y$, all RD-factors $A_{C}$ are parts of the bottom shape $S t_{k}^{1}$, except for the first and the last RD-factors: there $A_{C}=Y_{r}$ or $A_{C}=\emptyset$. In any case, none of the upper shapes $S t_{k}^{2}, S t_{k}^{3}, \ldots$ are broken up in (11). Thus, by induction hypothesis, we can apply $\sigma$ and $\vec{t}$ to each $Y_{C}:\left(Y_{C}^{\sigma}\right)^{\vec{t}}=A_{C} \times\left(B_{C}^{\sigma}\right)^{\vec{t}}$. We can then put back together the resulting diagrams via another application of the row-decomposition 
formula:

$$
Y \equiv \sum_{C} Y_{C}=\sum_{C} A_{C} \times B_{C} \stackrel{\text { ind. }}{\equiv} \sum_{C} A_{C} \times\left(B_{C}^{\sigma}\right)^{\vec{t}} \equiv\left(Y^{\sigma}\right)^{\vec{t}} .
$$

This shows that leaving the bottom shape fixed, we can permute and transpose the upper shapes in any way we like. We also know, again from Theorem 1, that transposing the whole diagram $Y$ yields a numerically equivalent diagram $Y^{t}$. It is an easy exercise in algebra to verify that these two types of operations generate the whole group of operations required in Corollary 2.

The conclusion of Corollary 2 holds under a slightly relaxed hypothesis regarding which staircases can be used instead of the critical staircase: as long as the RD (or CD) formula breaks up only the bottom (or only the top) hanging shape, the above proof goes through without modifications. Further generalizations are also possible, for instance, applying recursively the Corollary just within a hanging shape. Finally, this can all be used to write down a generating function for the numbers $T_{(213)}(Y)$, but we will not do this here since it will take us too far a field.

\section{Further discussion}

A careful investigation of the new Wilf-pair (546213) (465213) in $S_{6}$ leads to the observation that both permutation matrices can be decomposed into two blocks of $3 \times 3$ matrices, and further, that moving from one decomposition to the other involves a transposition of one of the blocks. Thus, one might be lead to conjecture that for any permutation matrices $A$ and $B$, the following permutation matrices are Wilf-equivalent:

$$
\left(\begin{array}{l|l}
A & 0 \\
\hline 0 & B
\end{array}\right) \sim\left(\begin{array}{l|l}
A^{t} & 0 \\
\hline 0 & B
\end{array}\right)
$$

In order for (12) to give any new Wilf-classes, other than those obtained by symmetry or $I_{t} \stackrel{s}{\sim} J_{t}$, both $A$ and $B$ must be non-symmetric matrices. In $S_{6}$, there is only one such pair up to symmetry (denote the 1's by dots, and omit all 0's):

$$
M(546213)=\left(\begin{array}{l|l}
\bullet & \\
\hline & \ddots
\end{array}\right) \sim\left(\begin{array}{l|l}
\bullet \cdot & \\
\hline & \ddots
\end{array}\right)=M(465213) .
$$

In $S_{7}$, there are essentially 7 new Wilf-pairs which are covered by (12). Not surprisingly, these are the pairs appearing in figure 7. One possible approach to prove (12) would be to show $A \stackrel{s}{\sim} A^{t}$ for any permutation matrix $A$. Unfortunately, this is not true; it fails already in $S_{4}$, e.g. (3142) $\stackrel{s}{\psi}(2413)$ since

$$
\left|S_{(6,6,6,6,5,5)}(3142)\right|=394<395=\left|S_{(6,6,6,6,5,5)}(2413)\right| .
$$




\begin{tabular}{|c||c|c|c|c|c|}
\hline \hline$\tau \sim \sigma$ in $S_{7}$ & $S_{9}(\tau)$ & $S_{10}(\tau)$ & $S_{11}(\tau)$ & $S_{12}(\tau)$ & $S_{13}(\tau)$ \\
\hline \hline$(6571342) \sim(5761342)$ & 361300 & 3587768 & 38951398 & 457416920 & 5756026177 \\
\hline$(6571423) \sim(5761423)$ & 361300 & 3587768 & 38951411 & 457418106 & 5756088993 \\
\hline$(6572413) \sim(5762413)$ & 361300 & 3587768 & 38951430 & 457419793 & 5756176230 \\
\hline$(6572431) \sim(5762431)$ & 361300 & 3587768 & 38951467 & 457423216 & 5756360170 \\
\hline$(6574132) \sim(5764132)$ & 361300 & 3587780 & 38952330 & 457459680 & 5757549454 \\
\hline$(6571432) \sim(5761432)$ & 361301 & 3587834 & 38953996 & 457496956 & 5758168203 \\
\hline$(6571243) \sim(5761243)$ & 361301 & 3587834 & 38954024 & 457499462 & 5758298471 \\
\hline
\end{tabular}

Figure 7. Final Wilf-equivalences in $S_{7}$.

The Wilf-equivalence in (12) also fails in $S_{8}$. For example:

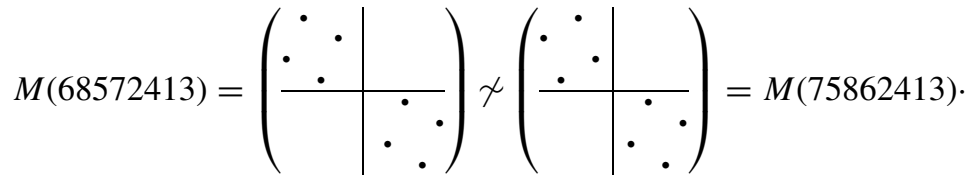

The two permutations are equinumerant up to level 11, but they split on level 12:

$$
\left|S_{12}(75862413)\right|=476576750<476576751=\left|S_{12}(68572413)\right| .
$$

This forces a reexamination of the 8 new Wilf-pairs in $S_{6}$ and $S_{7}$. If we choose the "right" representatives of the symmetry classes, we can see that each permutation matrix contains a block corresponding to (213) or (132). This led to conjecturing and proving the shapeWilf-equivalence $(213) \stackrel{s}{\sim}(132)$. As we can see, this SWE is far from coincidental, and it is the reason for the infinitely many new Wilf-equivalences of Theorem 1.

Let us now shift the emphasis of our discussion to a slightly different question. If we write down a table enumerating $\left|S_{n}(\tau)\right|$ for $\tau \in S_{4}$ as $n$ increases, we notice a very plausible long-standing conjecture, which was first mentioned in [18] as a question:

Question 1 Is it true that if $\left|S_{k}(\tau)\right|<\left|S_{k}(\sigma)\right|$ for some $k$, then $\left|S_{n}(\tau)\right|<\left|S_{n}(\sigma)\right|$ for all $n \geq k$ ? In other words, modulo Wilf-equivalence, can we order linearly all permutations in $S_{n}$ according to their relative restrictiveness: $\tau<\sigma$ if $\left|S_{k}(\tau)\right|<\left|S_{k}(\sigma)\right|$ for some $k$ ?

$S_{4}$ is the first non-trivial case of Question 1. It was partially answered positively by Bóna in [4], where he shows $\left|S_{n}(1423)\right|<\left|S_{n}(1324)\right|$ for $n \geq 6$ and $\left|S_{n}(1234)\right|<\left|S_{n}(1324)\right|$ for $n \geq 7$, in relation to a conjecture of Wilf and Stanley. To the best of our knowledge, no one had published a counterexample to Question 1, until we found a counterexample in $S_{5}$, followed by various types of counterexamples in $S_{6}$ and $S_{7}$.

As figure 8 suggests, (53241) and (43251) cannot be ordered since $S_{k}(53241)<S_{k}(43251)$ for $k \leq 12$, but $S_{13}(53241)>S_{13}(43251)$. Figures 9 and 10 list all counterexamples up to level 13 in $S_{6}$, and some counterexamples in $S_{7}$. The asterisks indicate the first level at which the corresponding permutations "switch" their relative restrictiveness, and hence cannot be ordered as in Conjecture 1. The "!!" in figure 9 refers to the permutation (546213), which is also part of the new Wilf-equivalence of Theorem 1. 


\begin{tabular}{|l||l|l|l|l|l|l|l|}
\hline$\tau \in S_{5}$ & $S_{7}(\tau)$ & $S_{8}(\tau)$ & $S_{9}(\tau)$ & $S_{10}(\tau)$ & $S_{11}(\tau)$ & $S_{12}(\tau)$ & $S_{13}(\tau)$ \\
\hline \hline$(25314)$ & 4578 & 33184 & 258757 & 2136978 & 18478134 & 165857600 & 1535336290 \\
\hline$(31524)$ & 4579 & 33216 & 259401 & 2147525 & 18632512 & 167969934 & 1563027614 \\
\hline$(35214)$ & 4579 & 33218 & 259483 & 2149558 & 18672277 & 168648090 & 1573625606 \\
\hline$(35124)$ & 4580 & 33249 & 260092 & 2159381 & 18815124 & 170605392 & 1599499163 \\
\hline$(53124)$ & 4580 & 33252 & 260202 & 2161837 & 18858720 & 171285237 & 1609282391 \\
\hline$(42351)$ & 4580 & 33252 & 260204 & 2161930 & 18861307 & 171341565 & 1610345257 \\
\hline$(35241)$ & 4580 & 33254 & 260285 & 2163930 & 18900534 & 172016256 & 1621031261 \\
\hline$(53241)$ & 4580 & 33256 & 260370 & 2166120 & 18945144 & 172810050 & $1633997788^{*}$ \\
\hline$(43251)$ & 4581 & 33283 & 260805 & 2171393 & 18994464 & 173094540 & $1632480259^{*}$ \\
\hline$(32541)$ & 4581 & 33284 & 260847 & 2172454 & 19015582 & 173461305 & 1638327423 \\
\hline$(34215)$ & 4581 & 33285 & 260886 & 2173374 & 19032746 & 173741467 & 1642533692 \\
\hline$(31245)$ & 4581 & 33286 & 260927 & 2174398 & 19053058 & 174094868 & 1648198050 \\
\hline$(42315)$ & 4581 & 33287 & 260967 & 2175379 & 19072271 & 174426353 & 1653484169 \\
\hline$(12345)$ & 4582 & 33324 & 261808 & 2190688 & 19318688 & 178108704 & 1705985883 \\
\hline$(53421)$ & 4582 & 33325 & 261853 & 2191902 & 19344408 & 178582940 & 1713999264 \\
\hline$(52341)$ & 4582 & 33325 & 261863 & 2192390 & 19358590 & 178904675 & 1720317763 \\
\hline
\end{tabular}

Figure 8. Classification of $S_{5}$ up to Wilf equivalence.

\begin{tabular}{|l||l|l|l|l|l|}
\hline \hline$r \in S_{7}$ & $S_{9}(\tau)$ & $S_{10}(\tau)$ & $S_{11}(\tau)$ & $S_{12}(\tau)$ & $S_{13}(\tau)$ \\
\hline \hline 253146 & 39424 & 344580 & 3283521 & $33633237^{*}$ & 366084190 \\
523614 & 39425 & 344611 & 3283955 & $33632674^{*}$ & 365858205 \\
\hline 352164 & 39425 & 344619 & 3284418 & $33648781^{*}$ & 366298292 \\
426153 & 39425 & 344620 & 3284441 & $33648549^{*}$ & 366268369 \\
\hline 365241 & 39425 & 344633 & 3285228 & $33676816^{*}$ & 367058930 \\
236145 & 39426 & 344661 & 3285505 & $33671423^{*}$ & 366717782 \\
\hline 356214 & 39426 & 344671 & 3286054 & 33689584 & $367192027^{*}$ \\
315642 & 39426 & 344672 & 3286086 & 33689894 & $367181171^{*}$ \\
\hline 315426 & 39426 & 344678 & 3286493 & $33705839^{*}$ & 367659357 \\
524316 & 39426 & 344679 & 3286521 & $33705830^{*}$ & 367633865 \\
\hline 325146 & 39426 & 344681 & 3286660 & $33711372^{*}$ & 367802282 \\
532416 & 39426 & 344682 & 3286686 & $33711239^{*}$ & 367772447 \\
\hline 326154 & 39427 & 344724 & 3287748 & $33732379^{*}$ & 368139497 \\
156324 & 39427 & 344724 & 3287749 & $33732314^{*}$ & 368131670 \\
\hline 643251 & 39427 & 344725 & 3287840 & $33736582^{*}$ & 368277055 \\
453261 & 39427 & 344726 & 3287851 & $33735408^{*}$ & 368204877 \\
\hline 435216 & 39427 & 344726 & 3287877 & 33737112 & $368270512^{*}$ \\
532164 & 39427 & 344727 & 3287904 & 33737204 & $368255863^{*}$ \\
\hline 632541 & 39427 & 344727 & $3287974^{*}$ & 33741840 & 368436494 \\
513246 & 39427 & 344728 & $3287971^{*}$ & 33739711 & 368325636 \\
\hline 624351 & 39427 & 344732 & 3288259 & 33751604 & $368699773^{*}$ \\
652413 & 39427 & 344733 & 3288292 & 33751853 & $368681504^{*}$ \\
\hline 643521 & 39427 & 344735 & 3288426 & 33757116 & $368841108^{*}$ \\
546213 & 39428 & 344772 & 3289163 & 33765743 & $368833207^{*} ! !$ \\
\hline \hline
\end{tabular}

Figure 9. Counterexamples to Question 1 in $S_{6}$.

\begin{tabular}{|c||c|l|l|l|l|}
\hline \hline$\tau \in S_{7}$ & $S_{9}(\tau)$ & $S_{10}(\tau)$ & $S_{11}(\tau)$ & $S_{12}(\tau)$ & $S_{13}(\tau)$ \\
\hline \hline 4725631 & 361297 & 3587548 & $38941876^{* *}$ & $457096663^{*}$ & 5746716800 \\
5427316 & 361297 & 3587549 & $38941876^{* *}$ & $457093463^{*}$ & 5746500592 \\
\hline 7536241 & 361297 & 3587572 & $38943767^{*}$ & 457184933 & 5749921907 \\
2645173 & 361298 & 3587605 & $38943703^{*}$ & 457138045 & 5747366258 \\
\hline
\end{tabular}

Figure 10. Some counterexamples to Question 1 in $S_{7}$. 


\begin{tabular}{|c||c|c|c|c|c|c|}
\hline \hline$\tau \in S_{6}$ & $S_{8}(\tau)$ & $S_{9}(\tau)$ & $S_{10}(\tau)$ & $S_{11}(\tau)$ & $S_{12}(\tau)$ & $S_{13}(\tau)$ \\
\hline \hline$(546213)$ & 39428 & 344772 & 3289163 & 33765743 & 368833207 & 4247979687 \\
\hline$(465213)$ & 39428 & 344772 & 3289163 & 33765743 & 368833207 & 4247979687 \\
\hline
\end{tabular}

Figure 11. Missing pair of Wilf-equivalence in $S_{6}$.

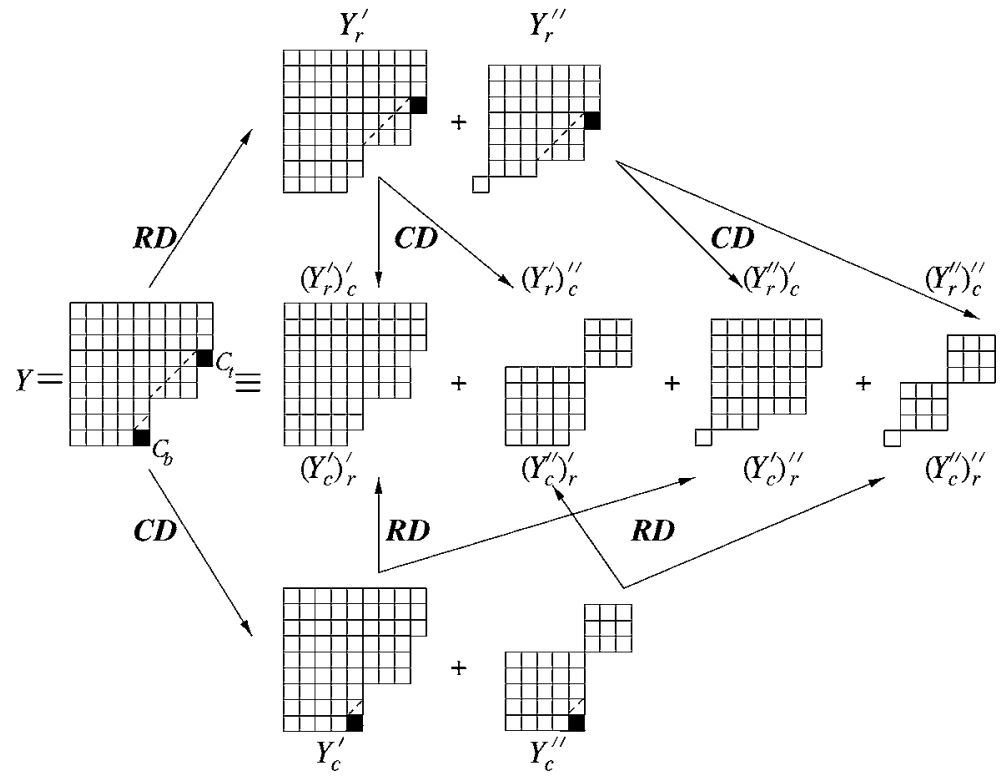

Figure 12. Case I for $Y(9,9,9,9,8,8,5,5,5)$.

However, the above counterexamples do not preclude an asymptotic ordering of all permutations in $S_{n}$.

Definition 12 For $\tau, \sigma \in S_{n}$, we say that $\tau$ is asymptotically smaller than $\sigma$ if $\left|S_{k}(\tau)\right|<$ $\left|S_{k}(\sigma)\right|$ for all $k \gg 0$.

Conjecture 1 We can order asymptotically all permutations in $S_{n}$, modulo Wilfequivalence.

Regev [12] and Bóna [5] have worked on asymptotic behavior of certain types of permutations, but as of now, Conjecture 1 and some possible modifications of it are far from proven.

Finally, we haven't observed examples of permutations "switching" their relative positions more than once. Thus, a stronger version of the above conjecture is possible, where assymptotic ordering is replaced by the previous usual ordering, allowing for one switch: $\tau<{ }_{1} \sigma$ if $\left|S_{n}(\tau) \geq\right| S_{n}(\sigma) \mid$ for $n \leq K$ and $\left|S_{n}(\tau) \leq\right| S_{n}(\sigma) \mid$ for $n>K$. Here $K$ depends on $\tau$ and $\sigma$, and if $K=0, \tau<\sigma$ in the strongest sense as in Question 1. 


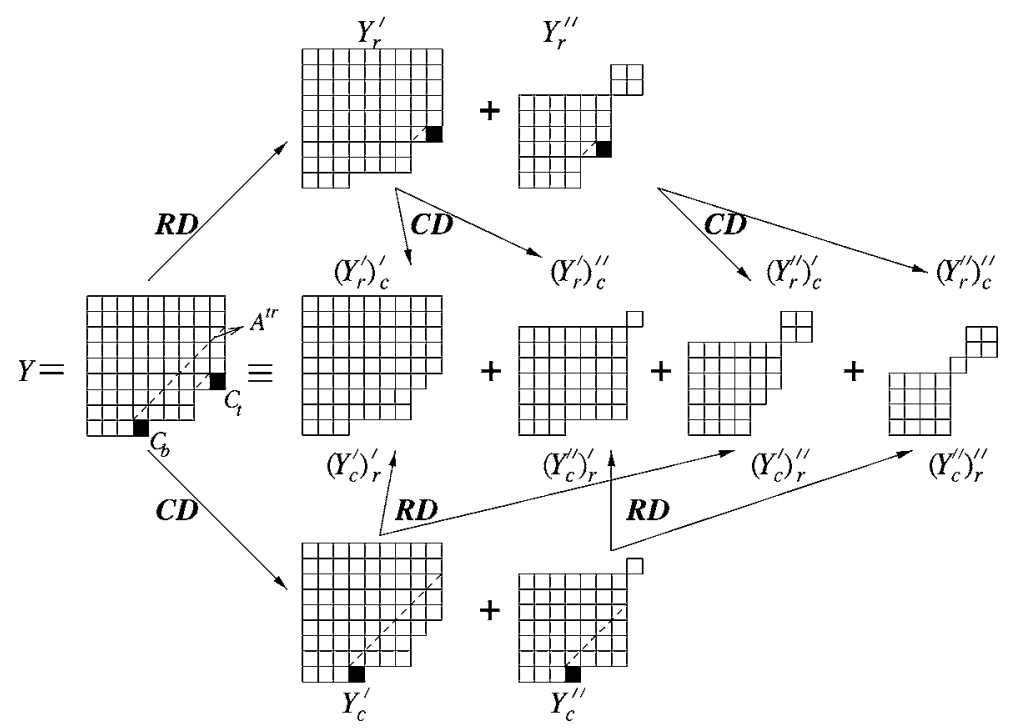

Figure 13. Case II for $Y(9,9,9,9,9,9,7,7,4)$.

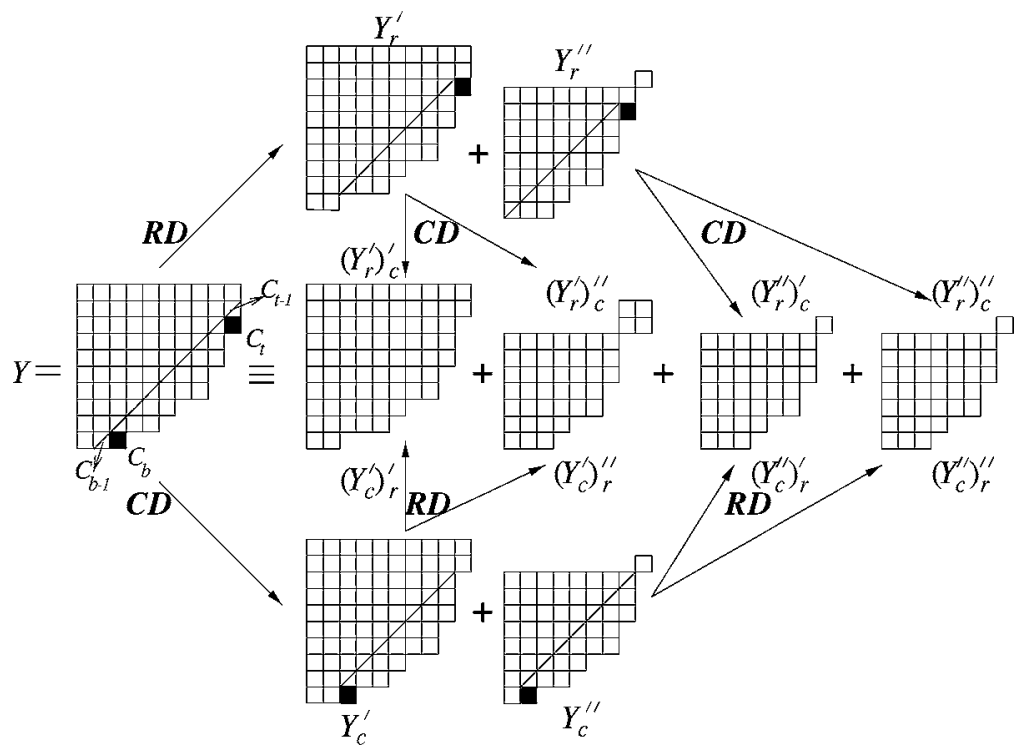

Figure 14. Case IV for $Y(10,10,10,9,9,8,8,6,5,3)$. 
Conjecture 2 We can order linearly the Wilf-classes in $S_{n}$ under the relation $<_{1}$.

\section{Acknowledgments}

Zvezdelina Stankova is grateful to Bernd Sturmfels (UC Berkeley) for his helpful suggestions both on mathematical and computational issues related to the project; to David Moews (University of Connecticut) for writing two of the computer programs used in this project; and to Paulo de Souza (UC Berkeley) and Tom Davis (Palo Alto) for their generous help in installing, testing and running the necessary computer software. Both authors would like to thank Olivier Guibert (Université Bordeaux 1, Talence Cedex, France) for providing his computer program on enumeration of forbidden subsequences, and to the referees for providing helpful suggestions.

\section{Notes}

1. To keep the resemblance with the "shape" of $\tau$, we coordinatize $M(\tau)$ from the bottom left corner.

2. In all tables, we skip the column corresponding to $\left|S_{n+1}(\tau)\right|$ if $\tau \in S_{n}$. It is easy to see that all permutations in $S_{n}$ are equinumerant on level $n+1$ (for example, cf. [11]).

\section{References}

1. E. Babson and J. West, “The permutations $123 p_{4} \ldots p_{t}$ and $321 p_{4} \ldots p_{t}$ are Wilf-equivalent," Graphs Combin. 16(4) (2000), 373-380.

2. J. Backelin, J. West, and G. Xin, "Wilf-equivalence for singleton classes," preprint.

3. S. Billey and G. Warrington, "Kashdan-Lusztig polynomials for 321-hexagon-avoiding permutations," arXiv:math.CO/0005052, 5 May 2000.

4. M. Bóna, "Permutations avoiding certain patterns. The case of length 4 and some generalizations," Discrete Math. 175 (1997), 55-67.

5. M. Bóna, "The solution of a conjecture of Wilf and Stanley for all layered patterns," J. Combin. Theory Ser. A, 85 (1999), 96-104.

6. D. Knuth, "Permutations, matrices, and generalized Young tableaux," Pacific J. of Math. 34 (1970), 709-727.

7. D. Knuth, The Art of Computer Programming, Vol. 3, Addison-Wesley, Reading, MA, 1973.

8. V. Lakshmibai and B. Sandhya, "Criterion for smoothness of Schubert varieties in SL(N)/B," Proc. Indian Acad. Sci. Math. Sci., 100 (1990), 45-52.

9. L. Lovász, Combinatorial Problems and Exercises, North-Holland, New York, 1979.

10. I. Macdonald, Notes on Schubert Polynomials, Universite du Quebec, 1991.

11. N. Ray and J. West, "Posets of matrices and permutations with forbidden subsequences," preprint.

12. A. Regev, "Asymptotic values for degrees associated with strips of Young diagrams," Advances in Math. 41 (1981), 115-136.

13. D. Richards, "Ballot sequences and restricted permutations," Arts Combinatoria 25 (1988), 83-86.

14. D. Rotem, "On correspondence between binary trees and a certain type of permutation," Information Processing Letters 4 (1975), 58-61.

15. R. Simion and F. Schmidt, "Restricted permutations," European J. Combin. 6 (1985), 383-406.

16. Z. Stankova, "Forbidden subsequences," Discrete Math. 132 (1994), 291-316.

17. Z. Stankova, "Classification of forbidden subsequences of length 4," European J. Combin. 17 (1996), 501-517.

18. J. West, "Permutations with forbidden subsequences and stack-sortable permutations," Ph.D. thesis, MIT, 1990.

19. J. West, "Generating trees and the Catalan and Schröder numbers," Discrete Math. 146 (1995), $247-262$.

20. J. West, "Generating trees and forbidden subsequences," Discrete Math. 157 (1996), 363-374. 